\title{
Picky Eating and Nutritional Status among Vietnamese Children Under Five Years of Age in Hue, Central Vietnam
}

\author{
Hoang Thi Bach Yen ${ }^{1}$, Le Thi Huong*2, Vo Van Thang ${ }^{3}$ and Hoang Anh Tien ${ }^{4}$ \\ ${ }^{1} \mathrm{PhD}$. Student, Hue University, Vietnam \\ ${ }^{2}$ Institute for Preventive Medicine and Public Health, Hanoi Medical University, Vietnam \\ ${ }^{3}$ Faculty of Public Health, Vietnam \\ ${ }^{4}$ Hue College of Medicine and Pharmacy, Vietnam \\ *Corresponding author: Le Thi Huong, Institute for Preventive Medicine and Public Health, Hanoi Medical University, Vietnam
}

\section{ARTICLE INFO}

Received: 㓞 March 05, 2019

Published: 慧 March 15, 2019

Citation: Hoang Thi Bach Yen, Le Thi Huong, Vo Van Thang, Hoang Anh Tien. Picky Eating and Nutritional Status among Vietnamese Children Under Five Years of Age in Hue, Central Vietnam. Biomed J Sci \& Tech Res 16(1)2019. BJSTR. MS.ID.002785.

Keywords: Picky eating; nutritional status; Vietnamese children under 5 years of age

Abbreviations: PES: Picky Eating Scale; SD: Standard Deviation; PPS: Probability Proportional to Size; WAZ: Weight-for-Age Z-Score; HAZ: Heightfor-Age Z-Score; WHZ: Weight-forHeight Z-Score; BMI: Body Mass Index

\section{ABSTRACT}

Background and objectives: Picky eating is the rejection of a number of foods and low intake in children and is linked to nutritional problems. This study aimed to describe the prevalence and characteristics of picky eating as well as to explore the relationship between picky eating and nutritional status among Vietnamese children under five years of age in Hue, central Vietnam.

Methods: Children under five years of age and their parents/caregivers were selected using a multistage sampling technique. The Picky Eating Scale (PES) previously developed in Vietnam was used with parents to estimate the prevalence of picky eaters. Using WHO Anthro software for nutritional status assessment. Statistical analyses were performed using SPSS 20.0. Data was reported as mean \pm standard deviation (SD), numbers and percentages. Comparisons between groups were tested for statistical significance using Fisher's exact 2-tailed tests or 2-tailed t tests for independent samples as appropriate. Values of $p<0.05$ were considered statistically significant.

Results: Seven hundred and seventy-two (772) children under five years of age were recruited. The mean age was $34.2 \pm 15.5$ months old. Half $(50.8 \%)$ of the children were male. Prevalence of picky eaters was $25.3 \%$. The most common signs of picky eating were eating less (63.6\%), mealtime lasting for too long (62.1\%), retaining food in the mouth for a long time (57.4\%), and pressure eating (45.1\%). Prevalence of underweight, stunting and wasting were $2.2 \%, 11.6 \%$ and $2.2 \%$, respectively. There were negative relationships between picky eating and nutritional status, especially stunting and wasting $(\mathrm{p}<0.01)$.

Conclusion: Picky eating was relatively prevalent among Vietnamese children under five years of age and might result in stunting and wasting.

\section{Background}

Eating is to meet the essential needs of human beings and to sustain life, growth and development. In every stage of life, a human being will have different energy and nutrient needs. For children, nutrition during the first years of life has important implications for development as well as quality of later life [1]. During growth stages, if essential nutrients are not met, permanent damage to tissues and organs can occur [2]. The most important stage of development is from newborn to five years old. This is the fastest period of weight gain and it is during this time that many organs, particularly and the central nervous and motor systems, become fully functioning. As this is also a period of high nutritional needs and susceptibility to diseases, therefore, children are at greatest risk of malnutrition. Malnutrition not only affects the physical but also mental health of the child and causes severe consequences for society [3]. Improper nutrition is a direct cause of malnutrition and micronutrient deficiencies such as vitamin $\mathrm{A}$, iron, iodine, zinc 
amongst others. In addition, lack of food or inappropriate eating behavior may contribute to dietary inadequacy and is a risk factor for malnutrition [4]. Insufficient food availability is an important cause of undernutrition, while the ability or willingness to consume available foods is another major factor [5].

Picky eating is "the unwillingness to eat familiar foods or try new foods, severe enough to interfere with daily routines to an extent that is problematic to the parent, child, or parent-child relationship" [6]. It is relatively common among infants and children, often causing anxiety for parents and caregivers. Picky eating is often linked to nutritional problems [7] and is also a risk factor for the development of an eating disorder [8]. The prevalence of picky eaters fluctuates, depending on the definition and assessment methods used. Literature reviews indicate that picky eating is prevalent among children, especially in high income countries. Estimated prevalence of picky eaters in high income countries was $21 \%$ among children aged 3 - 4 years old in USA by Jacobi C [9]; $19 \%$ to $50 \%$ among children aged 4 - 24 months old by Carruth [10]; $44.6 \%$ among toddlers 12 - 47.9 months old by Klazine van der Horst [11]; 14-17\% among pre-schoolers in Canada by Dubois L [12]; 31\% in Australia by Rebecca Byrne [13]; 39\% of children 12-72 months old in Turkey by Orun E [14]; while Charlotte M Wright (2007) found a much lower prevalence in United Kingdom (8\%) [15].

There is some information on picky eating in Asia. The prevalence of picky eating in Singaporean children aged 1-10 years was $49.2 \%$ [16] and as high as 54\% in Taiwanese children aged 2-4 years [17]. A study implemented in urban areas of China showed that $23.8 \%$ of parents considered their infant or toddler to be a picky eater; the prevalence of picky eaters increased significantly with age, from $12.3 \%$ at $6-11$ months to $21.9 \%$ at $12-23$ months, and then to $36.1 \%$ at $24-35$ months $(\mathrm{p}<0.001)$ [18]. Another study in China showed a prevalence of picky eating as high as $54 \%$ among pre-schoolers. In Vietnam, studies on picky eating are very limited. Prevalence of picky eating among children under five years of age at the National Hospital of Pediatrics (Hanoi) was 44.9\% [19] and $20.8 \%$ was reported by a study in Ho Chi Minh City [20]. The prevalence was as high as $54.58 \%$ among children from 1 - 6 years old in Ho Chi Minh City [21]. As Vietnam undergoes a transition in nutrition, picky eating is anticipated to become an emerging issue for children when access to stable food supplies are guaranteed. Having a scale to define picky eating in the Vietnamese context is essential not only for the child, parents, caregivers but also for health and educational workers. This study aimed to describe the prevalence and characteristics of picky eating and to explore the relationship between picky eating and nutritional status among children under five years of age living in Hue, central Vietnam.

\section{Methodology}

\section{Participants}

Children under five year of age living in Hue, central Vietnam and their parents or caregivers.

\section{Methods}

Study Design: The picky eating study was a three phases study in which phase 1 was an explanatory study for developing and piloting the tools. Phase 2 included 2 stages: the first stage was a cross-sectional study aimed to estimate the prevalence and to describe some characteristics of picky eating as well as nutritional status of children, while the second stage was a qualitative research which included 10 in-depth interviews and two focus group discussions aimed to complement quantitative results. Phase 3 was a case-control study aimed to determine risk factors of picky eating. Refer to Figure 1 for the flow chart of the study. The present analysis used data from the baseline measures of stage 1 from phase 2. A cross-sectional study was conducted on a sample size of 772 children under five years of age living in Hue city of central Vietnam. Questionnaires were used for face-to-face interviews with parents or caregivers about child feeding and eating behaviours. Height/length and weight of the child were obtained by trained researchers, following recommendations of the Vietnamese National Institute of Nutrition [22]. Data on height/length and weight of the children was used for nutritional status assessment by applying WHO Anthro software [23].

Sampling Method: The multistage sampling method was used for randomly selecting a sample of 4 out of 27 wards of Hue city. Based on the list of children under 5 years of age given by each commune health center, we calculated the total number of children in all four wards, then calculated the number of children for each ward by probability proportional to size (PPS) technique. In each ward, random sampling was used to choose children for the final sample.

Inclusion and Exclusion Criteria: Children were considered for inclusion if they were under 5 years old at the time of the study, were living in the study area at the time of the study and father, mother or caregivers agreed to participate (verbal consent). Children were excluded from the study if they had abnormalities of the mouth that might affect their ability to eat or other special conditions, for example Down syndrome, mental retardation (with medical record); or their father, mother; or caregivers did not agree to participate.

\section{Assessment Criteria}

Picky eating: The Picky Eating Scale (PES) had previously been developed after a review of the literature (phase 1) and by observing the main meals of ten Vietnamese children under five years of age, considered to be picky eaters by parents/caregivers living in Hue, Vietnam. Observations occurred at participants' houses or public places such as parks or food retail outlets between October and November of 2014. The types and amounts of food consumed, as well as the length of each mealtime, eating and playing activities, attitudes and emotions of both the child and feeding person were recorded. All eating activities of children were grouped into three main themes based on the observations and literature: length of 
time for each meal and eating activities of the children (eating, chewing, holding food); number of meals, diversity and amount of food that the child consumed per day; emotional or behaviours (happy, sad, worried) of the child at mealtime. The Picky Eating Scale (PES) was developed by deploying aspects derived from these three main themes.

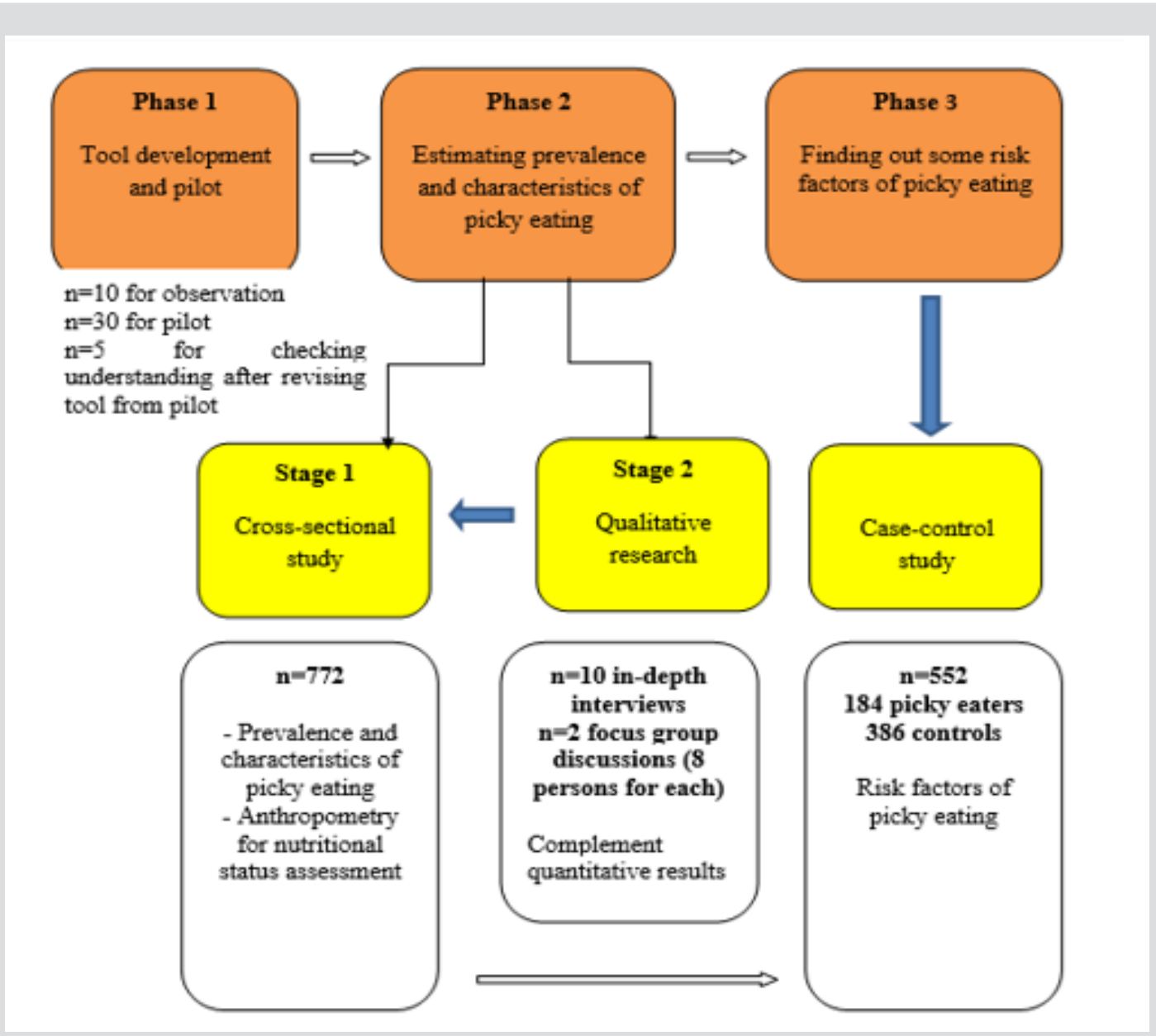

Figure 1: Flow chart of the study.

The scale had 14 questions, each question was scored based on the level of feeding difficulty, ranging from 0 to 3 points (the higher the score, the greater the picky eating behavior). The scoring from 0 to 3 points based on a previous study implemented in Vietnamese context [24]. In June 2017, two experienced nutrition staff from the Hue University of Medicine and Pharmacy conducted a pilot study on 30 parents/caregivers of children under five years of age, using face to face interviewing techniques. Comprehension of the questions was assessed, and questions were reworded to improve understanding. Cronbach's Alpha value was calculated for testing internal consistency of the PES. Results showed the value for Cronbach's Alpha of 0.82. PES was based on parent-report and provided a range of scores ( 0 to 42 ), with the used as the cut-off point for defining pickiness. As data analysis showed 10.5 as median value, children with a total score above 10.5 were defined as picky eaters.

Assessment of Nutritional Status: WHO Anthro software was used for assessment of nutritional status of children. The threshold for being assessed as undernutrition was <-2SD. a) Children with weight-for-age z-score (WAZ) of under -2SD would be classified as underweight and under -3SD would be classified as severely underweight

b) Children with length or height-for-age z-score (HAZ) of under -2SD would be classified as stunting and under -3SD would be classified as severely stunted

c) Children with weight-for-length and weight-for-height z-score (WHZ) of under-2SD would be classified as wasting and under -3SD would be classified as severely stunted. Children would be overweight or obese if WHZ was higher than +2SD [23].

\section{Statistical Analysis}

Statistical analyses were performed using SPSS (Statistical Product and Services Solutions, version 20.0). Continuous data were reported as mean \pm standard deviation (SD); ordinal data was reported as numbers and percentages. Comparisons between 
groups were tested for statistical significance using Fisher's exact 2-tailed tests or 2-tailed t tests for independent samples as appropriate. Values of $\mathrm{p}<0.05$ were considered statistically significant.

\section{Ethics Approval and Consent to Participate}

The aims of the research were explained to all parents or caregivers. Verbal informed consent was obtained prior to the interview, with participants signing the interview paper at the completion of the interview. For the qualitative component, attendants were asked to give permission prior to recording. All procedures were approved by the Committee for assessment of PhD proposal, Faculty of Public Health, Hue College of Medicine and Pharmacy, Hue University.

\section{Results}

\section{Characteristics of Children and Interviewees (Parents/ Caregivers)}

The study consisted of 772 children with a mean age of 34.2 \pm 15.5 months. Half $(50.8 \%)$ of the children were male and $79.1 \%$ were the first or second child of the family. As there were some families with more than one child in the study, only 716 parents or caregivers were interviewed. The mean age of interviewees was $37.5 \pm 12.2$ years old, $67.6 \%$ of them were mothers. Of the interviewees, $76.6 \%$ of had a level of education of secondary school and above, however, $2.2 \%$ of were illiteracy. The poor and the near poor accounted for $4.7 \%$ of the households.

Table 1: Number of meals per day.

\section{Picky Eating and Nutritional Status of Children}

\section{Picky Eating}

Prevalence of Picky Eaters: Prevalence of picky eaters was $25.3 \%$, the highest prevalence (31.3\%) was seen in group of under 24 months old. Prevalence of picky eating among boys and girls was $52.8 \%$ and $47.2 \%$, respectively, with no statistically significant difference between genders.

Common Signs of Picky Eating: The most common signs of picky eating were eating less (63.6\%), eating slowly, mealtime lasting for over 30 minutes (62.1\%), retaining food in the mouth (57.4\%), difficulty in feeding the child, pressure eating $(45.1 \%)$. $11.3 \%$ of children cried or had tantrums at mealtimes.

Onset Time of Picky Eating: There were $33.6 \%$ and $30.8 \%$ of picky eaters had signs at $12-<24$ months old and $6-<12$ months old, respectively. The prevalence decreased as the child got older with only $2.5 \%$ at 48 months and older being classified as picky eaters.

Number of Meals Per Day: Prevalence of picky eaters who ate not more than 3 meals per day (11.3\%) was higher than that of nonpicky eaters (3.8\%). There was a statistically significant difference regarding the number of meals per day between picky eaters and non-picky eaters $(p<0.001)$ but no statistically significant difference in terms of mean meals ( $p>0.05$ ) (Table 1).

\begin{tabular}{|c|c|c|c|c|c|}
\hline \multicolumn{6}{|c|}{ Number of Meals per Day } \\
\hline \multirow{2}{*}{ Number of meals per day } & \multicolumn{2}{|c|}{ Picky Eaters } & \multicolumn{2}{|c|}{ Non-Picky Eaters } & \multirow{2}{*}{$\chi^{2}, p$ value } \\
\hline & $\mathbf{n}$ & $\%$ & $\mathbf{n}$ & $\%$ & \\
\hline$\leq 3$ meals & 22 & 11.3 & 22 & 3.8 & \multirow{4}{*}{$\begin{array}{l}\chi^{2}=15.44 \\
p<0.001\end{array}$} \\
\hline 4-6 meals & 163 & 83.6 & 516 & 89.4 & \\
\hline$>6$ meals & 10 & 5.1 & 39 & 6.8 & \\
\hline Total & 195 & 100.0 & 577 & 100.0 & \\
\hline $\mathrm{X} \pm \mathrm{SD}$ (meals) & \multicolumn{2}{|c|}{$4.96 \pm 1.16$} & \multicolumn{2}{|c|}{$4.99 \pm 1.01$} & $>0.05$ \\
\hline
\end{tabular}

Nutritional Status: The prevalence of underweight, stunting and wasting among children was $2.2 \%, 11.7 \%$ and $2.2 \%$, respectively (see Table 2). In general, WAZ, HAZ and WHZ of the sample were lower and smaller compared to WHO Child Growth Standards (see Figure 2).

Table 2: Nutritional status of children.

\begin{tabular}{|c|c|c|}
\hline \multicolumn{3}{|c|}{ Nutritional Status of Children } \\
\hline Nutritional status & $\mathbf{n}$ & $\%$ \\
\hline \multicolumn{3}{|c|}{ Weight - for- age Z-score } \\
\hline Severe underweight & 3 & 0.4 \\
\hline Underweight & 14 & 1.8 \\
\hline Not underweight & 755 & 97.8 \\
\hline
\end{tabular}

\begin{tabular}{|c|c|c|}
\hline \multicolumn{3}{|c|}{ Length or height-for-age Z-score } \\
\hline Severe stunting & 31 & 4.0 \\
\hline Stunting & 59 & 7.7 \\
\hline Not stunting & 682 & 88.3 \\
\hline Weight-for-length and weight-for-height Z-score \\
\hline Severe wasting & 5 & 0.6 \\
\hline Wasting & 12 & 83.6 \\
\hline Normal & 647 & 14.0 \\
\hline Overweight or obese & 108 & 100.0 \\
\hline Total & 772 & \\
\hline
\end{tabular}




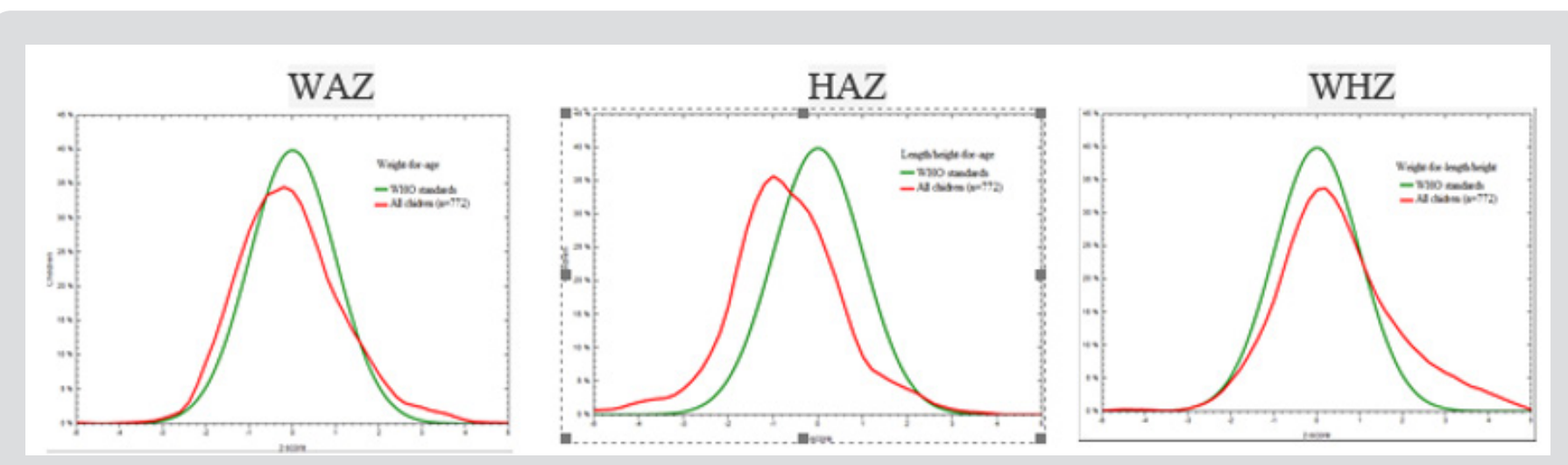

Figure 2: Nutritional status classified by three indicators included WAZ, HAZ and WHZ of the sample compared to WHO Child Growth Standards.

Relationship between Picky Eating and Nutritional Status: A negative relationship between picky eating and stunting $\left(\chi^{2}=\right.$ 5.721, $\mathrm{p}=0.017)$ and wasting $\left(\chi^{2}=10.428, \mathrm{p}=0.005\right)$ was found.

\section{Discussion}

Picky eating is relatively common among infants and children, often causing anxiety for parents and caregivers. At present, as there is no consistent definition of picky eating, there is no unified and well-defined method of assessment [6]. Several different measures have been developed to assess picky eating, ranging from a simple single question ("Is your child a picky eater?" [10,25]) to more complex multi-item sub-scales in larger questionnaires, generally related to eating behaviours [7,25-28]. Due to different assessment methods, prevalence of picky eating is variable, ranging from 20 to $60 \%$ in all children [29]. This study used a scale to define pickiness among Vietnamese children under five years of age. The scale was developed from three themes derived from observations and the scoring of the scale was quite consistent with the study by Huynh Van Son, implemented in the same country [21]. Prevalence of picky eaters in our study was $25.3 \%$, much lower than prevalence's reported from previous studies with only one question "Is your child a picky eater?" [10,25].

In a study of Hendy, Children's Eating Behaviour Questionnaire (Wardle) was used for interviewing and the mean was used as the cut-off point for defining picky eating [30]. Due to the small sample size and the abnormal distribution of data, we used the median as the cut-off point for defining pickiness. Our study showed a negative relationship between picky eating and HAZ as well as WHZ but no statistical relationship between picky eating and underweight. Picky eaters were more stunted and/or wasted compared to nonpicky eaters. However, information from Figure 2 showed that all three indicators of the studied children were lower than the reference population of the World Health Organization (lower peak and shift to the left, especially HAZ). Prevalence of overweight and obesity by WHZ was quite high (14.0\%). Some studies had found picky eating as a risk factor of underweight [12,31,32]. Some studies also showed that at the age of four, picky eaters had lower body mass index (BMI) and were more likely to be underweight than non-picky eaters $[12,26,31]$.

A longitudinal study found that prevalence of underweight among picky eaters was 20.6\%, higher than non-picky eaters [12]. Dubois conducted a longitudinal study on 1498 children and found that picky eaters were twice as likely to be at risk of being underweight at the age of 4.5 compared to non-picky eaters [31]. Ekstein Sivan found similar results that children with picky eating habits, especially those younger than 3 years of age, were at increased risk of being underweight. These are significant findings, as being underweight is an important risk factor for poor cognitive development, learning disabilities, long-term behavioural problems, increased prevalence and severity of infection, and high mortality rates [31]. Young Xue conducted a study on growth and development of 937 healthy preschool children in China and reported that picky eating behavior lasting over two years was associated with lower weight for age [32]. The question for the opposite hypothesis was if there was relationship between picky eating and weight gain. Galloway reported that picky eaters were less likely to be overweight. They consumed fewer fruits and vegetables, but also fewer fats and sweets. All girls consumed low amounts of vitamin E, calcium, and magnesium, but more picky girls were at risk for not meeting recommendations for vitamins $\mathrm{E}$ and $\mathrm{C}$ and also consumed significantly less fiber [26]. In contrast, according to Fisher $\mathrm{J} \mathrm{O}$ eating a limited amount of fruit and vegetables, but lots of foods with high-fat and energy can increase risk of overweight in picky eaters [33]. Furthermore, evidence is available that picky children may consume more sweetened foods [21], consequently, there is a risk that such children may establish a habit of over-consumption of energy dense, preferable foods, eventually culminating in excessive weight gain.

However, there are no longitudinal data to support this to date [34]. In addition, some studies observed no significant effects of picky eating on growth $[15,25]$. Paige K Berger conducted a lon- 
gitudinal study for 10 years on girls' picky eating in childhood. Results showed that persistent picky eaters were within the normal weight range, were less likely to be overweight, and had similar fruit intakes to those of non-picky eaters [35]. Wright conducted a cross-sectional analysis of data from a United Kingdom population-based birth cohort, which included 455 questionnaires completed by parents when their children were aged 30 months. Picky children described by parents were slightly lighter and shorter and had grown less well than the remainder, but these differences did not reach significance at the 0.05 level [15]. Picky children consumed fewer micronutrient-rich foods, such as fruits, vegetables, and meats [7] as well as low in vitamin C, vitamin E, fiber and folate foods $[26,34]$, which might result in the risk of undernutrition. However, regardless of whether the child is picky or not, the growth and development of the child's height is still a matter of controversy. Some studies focused on pre-schoolers with eating problems showed that picky eating had long-term problems associated with growth deficiency [31]. Other studies showed that pre-schoolers with picky behaviours were more likely to be underweight, were less likely to be overweight and were associated with an inadequate/suboptimal intake of essential nutrients $[12,26]$.

\section{Conclusion}

Picky eaters were relatively prevalent among Vietnamese children under five years of age. Picky eaters were more stunted and/or wasted compared to non-picky eaters.

\section{Conflicts of Interest}

This study has identified a scale for defining picky eating in children under five. However, further studies should be conducted to test the application of the scale. This study also found some characteristics of picky eating and its relationship to nutritional status. Identifying and understanding picky eating amongst children is valuable for parents, caregivers and health workers in order to create awareness regarding other aspects to prevent undernutrition.

\section{Funding Statement}

This work is a part of $\mathrm{PhD}$. research of the first author at Hue College of Medicine and Pharmacy, Hue University, Vietnam and receives no funding from any organization.

\section{Acknowledgement}

We would like to express our sincere thanks to all the participants involved in the study, especially the children and their caregivers for their coordination and support when conducting research.

\section{References}

1. Nguyen Cong Khan (2006) A new perspective on nurturing infants and young children according to WHO / UNICEF global recommendations. Nutrition \& Food Journal 2(2): 1-5.

2. Carroll Lutz, Karen Przytulski (2006) Life cycle nutrition "Pregnancy and lactation", in Nutrition and diet therapy: Evidence-based applications. Philadelphia, pp. 201-222.

3. (1998) Ministry of Health, Institute of Nutrition Guidelines for assessing the status of nutrition and food in a community. Hanoi Medical Publishing House, p. 39,61,68-71.
4. Ellen Frances Furman (2012) The Theory of Compromised Eating Behavior, Doctor of Philosophy, School of Nursing University of Massachusetts - Amherst, USA.

5. Maurice E Shils, Moshe Shike, A Catharine Ross, Benjamin Caballero, Robert J Cousins (2006) Pediatric feeding problems, in Modern nutrition in health and disease Lippincott Williams \& Wilkins: Philadelphia, pp. 875-880.

6. Caroline M Taylor, Susan M Wernimont, Kate Northstone, Pauline M Emmett (2015) Picky/fussy eating in children: Review of definitions, assessment, prevalence and dietary intakes. Appetite 95: 349-359.

7. Shim J E, Kim JM, Mathai RA (2011) Associations of infant feeding practices and picky eating behaviors of preschool children. J Am Diet Assoc 111(9): 1363-1368.

8. Melanie A Fernandez (2013) Picky Eater or Eating Disorder? Instructor 122(6): 41.

9. Jacobi C Agras WS, Bryson S (2003) Behavioral validation, precursors and concomitants of picky eating in childhood. Journal of American Academy of Child and Adolescent Psychiatry 42(1): 76-84.

10. Carruth BR, Ziegler Paula J, Gordon Anne, Barr Susan I (2004) Prevalence of picky eaters among infants and toddlers and their caregivers' decisions about offering a new food. Journal of the American Dietetic Association 104(S1): 57-64.

11. Klazine van der Horst, Denise M Deming, Ruta Lesniauskas, B Thomas Carr, Kathleen C Reidy (2016) Picky eating: Associations with child eating characteristics and food intake. Appetite 103: 286-293.

12. Dubois L, Farmer Anna, Girard Manon, Peterson Kelly, Tatone Tokuda Fabiola (2007) Problem eating behaviours related to social factors and body weight in preschool children: A longitudinal study, The international journal of Behavioral nutrition and physical activity 4(1):

13. Rebecca Byrne, Elena Jansen, Lynne Daniels (2017) Perceived fussy eating in Australian children at 14 months of age and subsequent use of maternal feeding practices at 2 years. International Journal of Behavioral Nutrition and Physical Activity 14(123): 1-9.

14. Orun E, Erdil Z, Cetinkaya S, Tufan N, Yalçin SS (2012) Problematic eating behavior in Turkish children aged 12-72 months: characteristics of mothers and children. Central Eur J Pub Health 20: 257-261.

15. Wright Charlotte, Parkinson M, Kathryn N, Shipton Deborah, Drewett Robert F (2007) How Do Toddler Eating Problems Relate to Their Eating Behavior, Food Preferences, and Growth? Pediatrics 120(4): 1069-1075.

16. Goh DY, Jacob A (2012) Perception of picky eating among children in Singapore and its impact on caregivers: a questionnaire survey. Asia Pacific family medicine 11(1): 5 .

17. Hsun-Chin Chao (2018) Association of picky eating with growth, nutritional status, development, physical activity and health in preschool children. Frontiers in Pediatrics 6: 22.

18. Ziyi Li Klazine van der Horst, Lisa R. Edelson Fries, Yu K, You L, Zhang Y, et al, (2017) Perceptions of food intake and weight status among parents of picky eating infants and toddlers in China: A cross-sectional study. Appetite 108: 456-463.

19. Nguyen Duc Tam, Luu Thi My Thuc (2017) The rate of anorexia of children $<5$ years old at the National Hospital of Pediatric Nutrition Clinic. Journal of Practical medicine 10(1060): 65-68.

20. Mai Thi My Thien, Vu Quynh Hoa, Do Thi Ngoc Diep, Tran Thi Minh Hanh (2014) Anorexia in children under 5 years old in Ho Chi Minh City, Journal of Nutrition and Food 10 (2): 40-46.

21. Huynh Van Son (2011) Manifestations of psychological anorexia in children from 1 to 6 years of age through the evaluation of parents. Science Journal of Ho Chi Minh City Pedagogical University 28: 23-32.

22. Hanoi Medical University (2016), Methods to evaluate nutritional status. Community Nutrition, Hanoi Medical Publishing House, pp. 193-218.

23. (2006) WHO Anthro 2005 Beta version Feb $17^{\text {th }}$, Software for assessing growth and development of the world's children. Geneva: WHO. 
24. Huynh Van Son, Vo Van Nam, Nguyen Hoang Khac Hieu (2012) Report summarizing the topic of scientific research and technology code CS2012.19.01.DATK "Experimental measures to overcome psychological anorexia of children aged 1 to 3 years".

25. Mascola AJ, Bryson SW, Agras WS (2010) Picky eating during childhood: a longitudinal study to age 11 years. Eating Behaviors 11(4): 253-257.

26. Amy T Galloway, Laura Fiorito, Yoonna Lee, Leann L Birch (2005) Parental pressure, dietary patterns, and weight status among girls who are "picky eaters". Journal of the American Dietetic Association 105 (4): 541-548.

27. Evans A, Seth J G, Smith S, Harris KK, Loyo J, et al. (2011) Parental feeding practices and concerns related to child underweight, picky eating, and using food to calm differ according to ethnicity/race, acculturation and income. Maternal and Child Health Journal 15(7): 899-909.

28. Jillian J Haszard, Paula ML Skidmore, Sheila M Williams, Rachael W Taylor (2015) Associations between parental feeding practices, problem food behaviours and dietary intake in New Zealand overweight children aged 4-8 years. Public Health Nutrition 18(6): 1036-1043.

29. Liliana Oliveros Leal, Rocío Ramos Paúl Salto, Luis Torres Cardona Jennifer A Williams, Barbara J Marriage, et al. (2012) Dietary Habits

ISSN: 2574-1241

DOI: 10.26717/BJSTR.2019.16.002785

Le Thi Huong. Biomed J Sci \& Tech Res

This work is licensed under Creative

Commons Attribution 4.0 License

Submission Link: https://biomedres.us/submit-manuscript.php and Nutrient Intakes of a Cohort of Healthy Children in Spain. The Open Nutrition Journal 6: 123-130.

30. Helen M Hendy Keith, E Williams, Katherine Riegel, Candace Paul (2010) Parent mealtime actions that mediate associations between children's fussy-eating and their weight and diet. Appetite 54(1): 191-195.

31. Ekstein Sivan, Laniado David, Glick Benjamin (2010) Does picky eating affect weight-for-length measurements in young children? Clinical pediatrics 49(3): 217-220.

32. Young Xue, Ai Zhao, Li Cai, Baoru Yang, Defu Ma, et al. (2015) Growth and Development in Chinese Pre-Schoolers with Picky Eating Behaviour: A Cross-Sectional Study, PloS One 10(4): e0123664.

33. Fisher JO, Birch LL (1995) Fat preferences and fat consumption of 3- to 5 -year-old children are related to parental adiposity. Journal of American Diet Association 95(7): 759-764.

34. Terence M Dovey, Paul A Staplesb, E Leigh Gibsonc, Jason CG Halfordd (2008) Food neophobia and 'picky/fussy' eating in children: A review. Appetite 50(2-3): 181-193.

35. Paige K Berger, Emily E Hohman, Michele E Marini, Savage JS, Birch LL (2016) Girls' picky eating in childhood is associated with normal weight status from ages 5 to $15 \mathrm{y}$. Am J Clin Nutr 104: 1577-1582.

$\begin{array}{ll}\text { BIOMEDICAL } & \text { Assets of Publishing with us } \\ \text { RESEARCHES } & \text { - Global archiving of articles } \\ & \text { - Immediate, unrestricted online access } \\ \end{array}$

